\title{
Correction to: The burden and management of anemia in Greek patients with inflammatory bowel disease: a retrospective, multicenter, observational study
}

\author{
Kalliopi Foteinogiannopoulou ${ }^{1 *}\left(\mathbb{0}\right.$, Konstantinos Karmiris $^{2}$, Georgios Axiaris ${ }^{3}$, Magdalini Velegraki ${ }^{2}$, \\ Antonios Gklavas ${ }^{4}$, Christina Kapizioni ${ }^{5}$, Charalabos Karageorgos ${ }^{6}$, Christina Kateri ${ }^{7}$, Anastasia Katsoula ${ }^{8}$, \\ Georgios Kokkotis ${ }^{9}$, Evgenia Koureta ${ }^{10}$, Charikleia Lamouri ${ }^{11}$, Panagiotis Markopoulos ${ }^{12}$, \\ Maria Palatianou ${ }^{13}$, Ploutarchos Pastras ${ }^{14}$, Konstantinos Fasoulas ${ }^{15}$, Olga Giouleme ${ }^{8}$, Evanthia Zampeli3, \\ Aggeliki Theodoropoulou ${ }^{2}$, Georgios Theocharis ${ }^{14}$, Konstantinos Thomopoulos ${ }^{14}$, Pantelis Karatzas ${ }^{10}$, \\ Konstantinos H. Katsanos ${ }^{11}$, Andreas Kapsoritakis ${ }^{7}$, Anastasia Kourikou ${ }^{6}$, Nikoleta Mathou ${ }^{16}$, \\ Spilios Manolakopoulos ${ }^{6}$, Georgios Michalopoulos ${ }^{5}$, Spyridon Michopoulos ${ }^{3}$, Alexandros Boubonaris ${ }^{17}$, \\ Giorgos Bamias ${ }^{9}$, Vasileios Papadopoulos ${ }^{7}$, George Papatheodoridis ${ }^{10}$, Ioannis Papaconstantinou ${ }^{4}$, \\ loannis Pachiadakis ${ }^{17}$, Konstantinos Soufleris ${ }^{15}$, Maria Tzouvala ${ }^{13}$, Christos Triantos $^{14}{ }^{14}$ Eftychia Tsironi $^{12}$, \\ Dimitrios K. Christodoulou ${ }^{11}$ and loannis E. Koutroubakis ${ }^{1}$ on behalf of the Hellenic group for the study of IBD
}

\section{Correction to: BMC Gastroenterol 21, 269 (2021).} https://doi.org/10.1186/s12876-021-01826-1

Following publication of the original article [1], it was reported that the affiliation of the corresponding author should be changed from "Dept. of Gastroenterology, University Hospital Heraklion, Heraklion, Crete, Greece" to "Gastroenterology Department, University Hospital of Heraklion, Medical School University of Crete, Greece".

The original article [1] has been corrected.

\section{Author details}

${ }^{1}$ Gastroenterology Department, University Hospital of Heraklion, Medical School University of Crete, P.O. BOX 1352, 71110 Heraklion, Crete, Greece. ${ }^{2}$ Department of Gastroenterology, Venizelio General Hospital, Heraklion, Greece. ${ }^{3}$ Department of Gastroenterology, General Hospital of Athens

The original article can be found online at https://doi.org/10.1186/s12876021-01826-1.

\section{*Correspondence: drfpopi@hotmail.com}

${ }^{1}$ Gastroenterology Department, University Hospital of Heraklion, Medical School University of Crete, P.O. BOX 1352, 71110 Heraklion, Crete, Greece Full list of author information is available at the end of the article

\begin{abstract}
"Alexandra", Athens, Greece. ${ }^{4}$ 2nd Department of Surgery, Aretaieion Hospital, National and Kapodistrian University of Athens, Athens, Greece. ${ }^{5}$ Department of Gastroenterology, General Hospital of Piraeus "Tzaneio", Athens, Greece. ${ }^{6}$ Hepato-Gastroenterology/Endoscopy Unit, 2nd Department of Internal Medicine, National and Kapodistrian University of Athens, Athens General Hospital "Heppocratio", Athens, Greece. 'Department of Gastroenterology, University General Hospital of Larissa, Larissa, Greece. ${ }^{8}$ 2nd Internal Medicine Department, General Hospital of Thessaloniki "Ippokratio", Thessaloniki, Greece. ${ }^{9}$ Gastroenterology Unit, 3rd Academic Department of Internal Medicine, National and Kapodistrian Univeristy of Athens, "Sotiria" General Hospital, Athens, Greece. ${ }^{10}$ Department of Gastroenterology, National and Kapodistrian University of Athens, General Hospital of Athens "Laiko", Athens, Greece. ${ }^{11}$ Department of Gastroenterology, University General Hospital of Ioannina, Ioannina, Greece. ${ }^{12}$ Department of Gastroenterology, "Metaxa” General Anticancer Hospital of Piraeus, Piraeus, Greece. ${ }^{13}$ Department of Gastroenterology, General Hospital of Nikaia Piraeus "Ag. Panteleimon"-General Hospital Dytikis Attikis "Agia Varvara", Athens, Greece. ${ }^{14}$ Division of Gastroenterology, Department of Internal Medicine, University Hospital of Patras, Patras, Greece. ${ }^{15}$ Department of Gastroenterology-Oncology, Theageneio Cancer Hospital of Thessaloniki, Thessaloniki, Greece. ${ }^{16}$ Department of Gastroenterology, General Hospital of Nea lonia "Konstantopoulio - Patision", Athens, Greece.

${ }^{17}$ Department of Gastroenterology, 424 General MilitaryHospital, Thessaloniki, Greece.
\end{abstract}

Published online: 29 July 2021

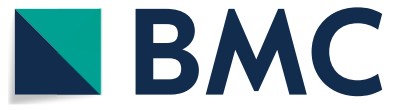

(c) The Author(s) 2021. This article is licensed under a Creative Commons Attribution 4.0 International License, which permits use, sharing, adaptation, distribution and reproduction in any medium or format, as long as you give appropriate credit to the original author(s) and the source, provide a link to the Creative Commons licence, and indicate if changes were made. The images or other third party material in this article are included in the article's Creative Commons licence, unless indicated otherwise in a credit line to the material. If material is not included in the article's Creative Commons licence and your intended use is not permitted by statutory regulation or exceeds the permitted use, you will need to obtain permission directly from the copyright holder. To view a copy of this licence, visit http://creativecommons.org/licenses/by/4.0/. The Creative Commons Public Domain Dedication waiver (http://creativecommons.org/publicdomain/zero/1.0/) applies to the data made available in this article, unless otherwise stated in a credit line to the data. 


\section{Reference}

1. Foteinogiannopoulou K, Karmiris K, Axiaris G, et al. The burden and management of anemia in Greek patients with inflammatory bowel disease: a retrospective, multicenter, observational study. BMC Gastroenterol. 2021;21:269. https://doi.org/10.1186/s12876-021-01826-1.

\section{Publisher's Note}

Springer Nature remains neutral with regard to jurisdictional claims in published maps and institutional affiliations. 